\title{
Validación de una escala de roles de víctimas y agresores asociados al acoso escolar
}

\section{González González Elsa Natalí ${ }^{1}$, Peña Ramos Martha Oli- via $^{2}$, Vera Noriega José Ángel ${ }^{3}$.}

Departamento de Desarrollo Humano y Bienestar Social, Centro de Investigación en Alimentación y Desarrollo A.C., Hermosillo.

\section{México}

Correspondencia: Elsa Natalí González González. Carretera a la Victoria Km .6 Ejido La Victoria, Apdo. Postal 1735, C.P. 83000, Hermosillo, Sonora. México. E-mail: lcengg@ gmail.com

(C) Education \& Psychology I+D+i and Ilustre Colegio Oficial de la Psicología de Andalucía Oriental (Spain) 


\section{Resumen}

Introducción. La escuela representa un espacio propicio en el que las interrelaciones entre los alumnos se conjugan en un mismo lugar, donde se aprenden reglas básicas de convivencia y socializacion mediante la adquisición y práctica de códigos socio-culturales. Sin embargo, estos aprendizajes se pueden ver mermados por situaciones de violencia escolar, ya que interfieren en los ambientes pacíficos que se pretenden crear para llevar a cabo labores de aprendizaje y convivencia, pudiendo llegar a degradar las relaciones de quienes participan e intervienen en esos espacios; Si bien, existen múltiples características de los actores que intervienen en este tipo de situaciones de violencia, los actores "primarios" son quienes agreden (agresores) y quienes reciben la agresión (víctimas), conformando una relación binaria entre sí, constituyéndose como el centro de la agresión.

Método. Basados en la teoría de respuesta al ítem mediante la calibración de reactivos por Rasch, Análisis Factorial Exploratorio y Confirmatorio, se validan las escalas de víctimas y agresores. Las medidas fueron aplicadas en secundarias públicas con jóvenes entre 12 y 17 años de edad; ambas conformadas por 12 ítems escalares tipo Likert que miden la percepción de los alumnos.

Resultados. Los parámetros psicométricos obtenidos para ambos modelos fueron idóneos. Los resultados muestran dos dimensiones en ambas escalas, en la de víctimas el sufrir: "violencia verbal/relacional" y "violencia física/social", mientras que la de agresores el infligir: "violencia verbal/física" y "violencia sexual/relacional".

Discusión y Conclusiones. Se encontró que ambas escalas coinciden en la eliminación de reactivos que tienen que ver con: violencia física hacia las pertenencias de otros, violencia sexual relacionada a tocar las partes íntimas de otro y violencia social referido al sometimiento de otros para hacer o dejar de hacer algo; se discute desde la concepción de la cultura del silencio. Se concluye que las escalas analizadas en conjunto tienen valores psicométricos adecuados para ser utilizadas en posteriores análisis.

Palabras Clave: Acoso escolar, Agresión, Educación secundaria, Víctimas 


\begin{abstract}
Introduction. The school is a suitable space in which the interrelationships between students come together in one place, where basic rules of coexistence and learning society through the acquisition and practice of socio-cultural codes. However, these learning can be seen undermined by situations of school violence and that interfere with the peaceful environment that are intended to create to carry out tasks of learning and coexistence, may reach degrade the relationships of those involved in those areas; While there are many characteristics of the actors involved in such situations of violence, the "primary" stakeholders are those who attack (aggressors) and who receives the aggression (victims), forming a binary relationship to each other, constitu-leaving the center of the attack.
\end{abstract}

Method. Based on the item response theory by Rasch calibration reagents, exploratory and confirmatory factor analysis. The scales of victims and aggressors are validated, which were measured in public middle school with young people aged 12 to 17 years old; both scales formed 12 Likert items that measure the perception of students.

Results. Psychometric parameters obtained for confirmatory model of victims are suitable. The results show two dimensions on both scales, in the suffering of victims, "verbal violence / relational" and "physical / social violence", while the aggressors "verbal violence / physical" and "sexual violence / relational".

Discussion and Conclusion. It was found that both scales match in removing reagents that deal with: physical violence against the property of others, sexual violence related to touch the private parts of other people and relational violence referred to submission of others to do or not to do something; It discussed from conception of the culture of silence. We conclude that the scales analyzed together are suitable for use in subsequent analyzes psychometric tests.

Keywords: Aggression, Bullying, Middle School, Victims. 


\section{Introducción}

Dos de los pilares básicos de la educación que son señalados por la UNESCO son el aprender a ser y el aprender a convivir. Sin embargo, las actividades curriculares que se basan en el aprender a conocer y en el aprender a hacer, se llevan más carga escolar, dejando un poco de lado aquello que tiene que ver con el ser y formar personas. Desde la niñez se van adquiriendo prácticas de socialización, por lo que la escuela representa un espacio propicio en el cual estas interrelaciones entre los alumnos, maestros, padres de familia, sociedad que le rodea, se conjugan en un mismo lugar, para aprender reglas básicas de convivencia democrática pacífica, mediante la adquisición y práctica de códigos socio-culturales.

Sin embargo, al relacionarse entre distintas personas, puede que se den conflictos, los cuales son normales en toda sociedad y en su mayoría surgen debido a la diversidad (diversidad entendiéndola como intereses, pensamientos, sentimientos, raza, cultura, distintos de los otros), pero de no saber cómo afrontar estos conflictos, pueden llegar a elevarse a niveles de violencia como el acoso escolar o bulying. Dicha violencia interfiere en los ambientes pacíficos que se pretenden crear para llevar a cabo labores de aprendizaje y convivencia, ya que degrada las relaciones de quienes participan e intervienen en esos espacios; como lo articulan Bourdieu y Passerson (1996) no todo es positivo, sino que se pueden llegar a producir y perpetuar mecanismos de dominación social que se ven reflejados en los diferentes tipos de violencia, incluyendo al acoso escolar y bullying.

Desde este punto, es necesario comprender que el acoso escolar o bullying refiere a relaciones en donde existe un desequilibrio de poder entre los estudiantes, relación que se mantiene a través del tiempo, es decir, es reiterativa, y tiene el objetivo de hacer daño a su contraparte, es decir, es intencional, y los que agreden tienen el conocimiento de que sus actos no son correctos. Existen diversas formas de agredir a otro no sólo físicamente, sino verbalmente (mediante apodos, insultos, etc.), socialmente (a través de la exclusión social), sexualmente (relaciones o tocamientos de índole sexual sin consentimiento), por medios electrónicos (a través de medios de comunicación, redes sociales, etc.), donde, sin importar la forma en que se agreda a las partes, siempre tendrán una cognotación de violencia psicológica que afecta a la víctima y al medio en que se desenvuelve a través de sus relaciones interpersonales. 
Si bien, existen múltiples características de los actores que intervienen en el acoso escolar, los "primarios" son quienes agreden (agresores) y quienes reciben la agresión (víctimas), ya que conforman una relación binaria entre sí, representando el centro de la agresión. Por un lado se encuentra la víctima, quien es acosada o victimizada cuando se siente coaccionada, degradada, humillada, abusada, intimidada, asustada, amenazada, o está sufriendo algún tipo de malestar emocional y/o físico ocasionado por uno o más de sus compañeros. Mientras que el agresor es quien empieza el ataque de forma reiterada contra su compañero (víctima), que consciente o inconscientemente éste sabe que al violentar a otros consigue un mayor nivel de poder dentro de las redes de socialización (Kulig, Hall \& Kalischuk, 2008). Los agresores presentan cuatro necesidades básicas según señala Rodríguez (2005) citado en Albaladejo (2011): necesidad del protagonismo, de sentir superioridad y poder, de ser diferentes y la necesidad de llenar un vacío emocional.

Las relaciones violentas entre agresores y víctimas desfavorecen el clima de aprendizaje pacífico deseable para el bienestar de los alumnos, debilitando la sana convivencia y los lazos relacionales entre los compañeros; lo que hace necesario conocer las conductas disruptivas que alteran la paz y los roles que se asumen en ese tipo de relaciones, ya que en los diferentes contextos sociales se encontraran diversos tipos de violencia, no siendo probables en todos ellos los mismos (Doménech e Íñiguez, 2002).

\section{Objetivo}

Por lo anterior, el objetivo de este trabajo se centra en validar la escala de víctimas y agresores y sus roles asociados al acoso escolar, midiendo las percepciones de los alumnos al protagonizar los roles de víctimas y agresores. Dicha validación se realiza por medio de un análisis psicométrico de perspectiva regional, con el fin de coadyuvar en la realización de análisis posteriores para el Estado de Sonora (México).

\section{Método}

\section{Participantes}

Se contó con la participación de 2345 estudiantes de secundaria públicas con edades comprendidas entre los 12 y 17 años de edad; el $48.53 \%$ de hombres, el 50.84\% de mujeres y un $0.63 \%$ que no contestaron el ítem de edad. Los niveles o grados educativos en secundarias 
públicas son $1^{\circ}, 2^{\circ}$ y $3^{\circ}$ contando con 937,717 y 691 alumnos respectivamente pertenecientes a alguna de las 64 escuelas secundarias de tipo federales y generales dentro del Estado de Sonora, México (Véase Tabla I).

Tabla 1. Distribución de alumnus por region del Estado de Sonora

\begin{tabular}{lcc}
\hline Municipio & $N$ & $\%$ \\
\hline Costa (Hermosillo) & 709 & 30,3 \\
Frontera (Caborca, Pitiquito, Agua Prieta) & 579 & 24,7 \\
$\begin{array}{l}\text { Sur (Obregón, Guaymas, Empalme, Na- } \\
\text { vojoa) }\end{array}$ & 783 & 33,4 \\
$\begin{array}{l}\text { Sierra (Mazatán, Sahuaripa, Moctezuma, } \\
\text { Huepac, Cumpas, Ures, Baviacora, Ra- }\end{array}$ & 274 & 11,6 \\
$\begin{array}{l}\text { yón, Carbó) } \\
\text { Total }\end{array}$ & \\
\hline
\end{tabular}

\section{Instrumentos}

El instrumento es un auto-informe que mide las percepciones de los alumnos en escalas con respuestas tipo Likert de cinco puntos, en las que se les preguntan las conductas de las que han sido participes y las que han sufrido (escala de agresores y víctimas respectivamente).

Escala de Agresores (Ortega y Del Rey, 2005), la cual evalúa la conducta agresiva hacia los compañeros en relación a un mes, desde sus diferentes dimensiones: físico, social, psicológico, sexual y cyber byllying.

Escala de tipos de acoso escolar o escala de víctimas (Valdés, 2013) recoge información referente a las conductas agresivas que reciben los alumnos por parte de sus compañeros desde sus diferentes dimensiones: físico, social, psicológico, sexual y cyber bullying.

Tomando en cuenta las medidas de Hair, Anderson, Tatham y Black (1999), el Alpha de Cronbach debe situarse por encima de 0.70 para ser aceptable. En la escala de agresores, este valor fue 0.84 , mientras que en la escala de víctimas se obtuvo 0.85, ajustándose así ambas escalas a una propuesta aceptable de " $\alpha$ ".

\section{Procedimiento}

Para acceder a las aulas de las escuelas se tuvo que realizar una exposición acerca de los objetivos de la investigación. Una vez conseguida la autorización de las personas corres- 
pondientes, se solicitó la autorización informada por escrito de los padres de los adolescentes que fueron seleccionados para participar en el estudio. Finalmente, se procuró la participación voluntaria de los estudiantes garantizándoles la confidencialidad de la información que ellos proporcionaron.

\section{Análisis Estadístico}

Referente al procedimiento para el análisis de las propiedades psicométricas del instrumento se establecieron evidencias de validez de constructo mediante una combinación de modelos estadísticos: Analisis de Rasch, Análisis factorial exploratorio y confirmatorio, además de análisis de fiabilidad, determinada mediante la consistencia interna de los puntajes. Para ello se ulizaron para la calibración de reactivos por Rasch, el programa Winsteps y para el Análisis de Confiabilidad, Factorial Exploratorio y Confirmatorio, se utilizó el procesador de datos SPSS y AMOS.

\section{Resultados}

Primeramente, se realizó la validez del constructo mediante un análisis de propiedades de los ítems, para lo cual se utilizó el modelo de crédito parcial de la teoría Rasch. Se encontró que los reactivos de las escalas de agresores y víctimas cumplen con los rangos propuestos, considerando que los estándares aceptables para el modelo de Rash, según Writht y Linacre (1994), se encuentran dentro de los rangos de normalidad aceptables (se ajustan a las normas esperadas) cuando los indicadores de Infit y Outfit tienen un rango entre 0.5 y 1.5, mientras que para el punto biserial (pbis), según Cháves y Saade (2009) los rangos son aceptables al ser mayores que 0.30, y finalmente la discriminación empírica (Disc) se encuentra dentro de normalidad (se ajustan a las normas esperadas) según González-Montesinos (2008) cuando el límite inferior es mayor de 0.90 debiendo estar lo más próximo a 1 (véase en Tabla 2 y 3 ).

Tabla 2. Estadísticos descriptvos. Resultado de la calibración de ítems para la escala de agresores.

\begin{tabular}{lllllll}
\hline Entry & Name & Measure & Infit & Outfit & Pbis & Disc \\
\hline R38 & Ignorarlos & 44.58 & 1.19 & 1.14 & 0.66 & 0.77 \\
R39 & No dejar que participen & 45.87 & 1.26 & 1.50 & 0.47 & 0.91 \\
R40 & Insultarlos & 42.65 & 1.14 & 1.01 & 0.64 & 0.92
\end{tabular}




\begin{tabular}{|c|c|c|c|c|c|c|}
\hline R41 & $\begin{array}{l}\text { Ponerles sobrenombres ofensi- } \\
\text { vos }\end{array}$ & 45.23 & 1.01 & 0.96 & 0.60 & 1.01 \\
\hline R42 & Esconderles las cosas & 43.74 & 0.99 & 0.99 & 0.59 & 0.97 \\
\hline $\mathrm{R} 44$ & Pegarles, patearlos, empujarlos & 46.54 & 0.98 & 0.91 & 0.58 & 1.03 \\
\hline R45 & Amenazarlos & 49.33 & 0.82 & 0.83 & 0.42 & 1.07 \\
\hline R47 & $\begin{array}{l}\text { Tocar las partes íntimas de un } \\
\text { compañero }\end{array}$ & 50.29 & 1.23 & 0.71 & 0.28 & 1.00 \\
\hline R48 & $\begin{array}{l}\text { Obligar a un compañero a que } \\
\text { te toque tus partes íntimas }\end{array}$ & 49.48 & 0.88 & 0.68 & 0.30 & 1.03 \\
\hline R49 & $\begin{array}{l}\text { Sacar del grupo de amigos a un } \\
\text { compañero }\end{array}$ & 47.02 & 1.14 & 1.50 & 0.44 & 0.94 \\
\hline $\mathrm{R} 50$ & $\begin{array}{l}\text { Dejar solo en actividades de } \\
\text { equipo a un compañero }\end{array}$ & 48.73 & 0.91 & 1.20 & 0.45 & 1.00 \\
\hline R51 & $\begin{array}{l}\text { Burlarse del físico de compañe- } \\
\text { ros }\end{array}$ & 48.32 & 0.91 & 0.84 & 0.51 & 1.05 \\
\hline R52 & $\begin{array}{l}\text { Agredirlo utilizando Facebook } \\
\text { y otro medio electrónico }\end{array}$ & 50.17 & 0.83 & 0.79 & 0.46 & 1.07 \\
\hline
\end{tabular}

Tabla 3. Estadísticos descriptivos. Resultado de la calibración de ítems para la escala de víctimas.

\begin{tabular}{|c|c|c|c|c|c|c|}
\hline Entry & Name & Measure & Infit & Outfit & Pbis & Disc \\
\hline R6 & Me ignoran & -0.22 & 0.99 & 0.92 & 0.52 & 0.99 \\
\hline R8 & Me insultan & -0.42 & 0.84 & 0.75 & 0.62 & 1.18 \\
\hline R9 & $\begin{array}{l}\text { Me ponen sobrenombres } \\
\text { ofensivos }\end{array}$ & -0.38 & 0.98 & 0.97 & 0.56 & 1.02 \\
\hline $\mathrm{R} 10$ & Hablan mal de mí & -0.53 & 0.99 & 1.01 & 0.56 & 0.96 \\
\hline R11 & Me esconden cosas & -0.06 & 1.03 & 1.01 & 0.50 & 0.95 \\
\hline R12 & Me roban cosas & -0.24 & 1.24 & 1.35 & 0.40 & 0.81 \\
\hline R13 & Me pegan & 0.11 & 0.89 & 0.92 & 0.57 & 1.04 \\
\hline R14 & $\begin{array}{l}\text { Me amenazan para que sien- } \\
\text { ta miedo }\end{array}$ & 0.38 & 0.75 & 0.54 & 0.62 & 1.12 \\
\hline R15 & $\begin{array}{l}\text { Me obligan a hacer cosas } \\
\text { que no quiero }\end{array}$ & 0.33 & 0.95 & 0.71 & 0.46 & 1.03 \\
\hline R16 & Me tocan mis partes íntimas & 0.49 & 1.04 & 0.84 & 0.39 & 1.00 \\
\hline R18 & $\begin{array}{l}\text { Me sacan del grupo de ami- } \\
\text { gos }\end{array}$ & 0.35 & 0.88 & 0.76 & 0.53 & 1.05 \\
\hline R19 & $\begin{array}{l}\text { Me dejan solo en actividades } \\
\text { de equipo }\end{array}$ & 0 & 0.91 & 0.83 & 0.56 & 1.05 \\
\hline $\mathrm{R} 20$ & Se burlan de mi físico & -0.07 & 0.96 & 0.81 & 0.54 & 1.05 \\
\hline
\end{tabular}


Sin embargo, para mejorar los estándares de la prueba, en la escala de agresores se decidió eliminar los reactivos 43 "robarles" y 46 "obligarles a hacer cosas que no quieren", debido a que no cumplían con datos aceptables que exige la normalidad del outfit (ver Tabla II).De igual manera, en la Escala de víctimas, se decidió eliminar los reactivos 7 "no me dejan participar" y 17 "me obligan a tocarles sus genitales" pues tampoco cumplían con los estándares aceptables de normalidad de outfit (ver Tabla 3).

En un segundo momento se procedió a realizar un análisis factorial por el método de extracción de máxima verosimilitud y de rotación Oblimin con Kaiser para cada una de las escalas. Se obtuvo un KMO (Kaiser-Meyer-Olkin) para la escala de agresores de 0.89 y para la escala de víctimas de 0.90, ambos encontrándose en rangos de normalidad (aceptables, esperados), siendo el límite inferior requerido de 0.70; así mismo se realizó una prueba de esfericidad de Bartlett que apoya la adecuación de solución factorial de $(* \mathrm{p}=0.00)$, apoyando así el procedimiento de la solución factorial, en la que se extrajeron dos factores en ambas escalas que explican en la escala de agresores el $32 \%$ de varianza total y en la escala de víctimas el $39 \%$ (véase en Tablas 4 y 5).

Tabla 4. Análisis factorial para la escala de agresores.

\begin{tabular}{rlrlc}
\hline Entry & Name & $F 1$ & $F 2$ & Comunalidad \\
\hline R40 & Insultarlos & $\mathbf{0 . 7 8 3}$ & -0.144 & 0.407 \\
R41 & Ponerles sobrenombres ofensivos & $\mathbf{0 . 7 1 1}$ & -0.043 & 0.396 \\
R42 & Esconderles las cosas & $\mathbf{0 . 5 8 5}$ & 0.037 & 0.315 \\
R38 & Ignorarlos & $\mathbf{0 . 5 7 4}$ & -0.017 & 0.280 \\
R44 & Pegarles, patearlos, empujarlos & $\mathbf{0 . 5 6 4}$ & 0.097 & 0.360 \\
R51 & Burlas del físico de compañeros & $\mathbf{0 . 4 5 0}$ & 0.255 & 0.363 \\
R45 & Amenazarlos & $\mathbf{0 . 3 6 0}$ & 0.312 & 0.341 \\
R39 & No dejar que participen & $\mathbf{0 . 3 5 4}$ & 0.191 & 0.220 \\
& Obligar a un compañero a que te to- & - & $\mathbf{0 . 7 9 3}$ & 0.380 \\
R48 & que tus partes íntimas & 0.120 & & \\
& Tocar las partes íntimas de un compa- & - & $\mathbf{0 . 5 9 3}$ & 0.276 \\
R47 & ñero & 0.054 & & \\
& Sacar del grupo de amigos a un com- & 0.157 & $\mathbf{0 . 5 3 9}$ & 0.361 \\
R49 & pañero & & & \multirow{2}{*}{$\begin{array}{l}\text { Agredirlos utilizando Facebook u otro } \\
\text { R52 }\end{array}$} \\
& medio electrónico & $\mathbf{0 . 5 0 7}$ & 0.372 \\
& Dejar solo en actividades de equipo a & & & \\
R50 & un compañero & $\mathbf{0 . 4 3 3}$ & 0.344 \\
\hline
\end{tabular}


Tabla 5. Análisis factorial para la escala de víctimas

\begin{tabular}{clccc} 
Entry & \multicolumn{1}{c}{ Name } & $F 1$ & $F 2$ & Comunalidad \\
\hline R8 & Me insultan & $\mathbf{0 . 7 6}$ & -0.41 & 0.479 \\
R9 & Me ponen sobrenombres ofensivos & $\mathbf{0 . 6 8}$ & -0.39 & 0.405 \\
R10 & Hablan mal de mí & $\mathbf{0 . 6 5}$ & -0.36 & 0.360 \\
R20 & Se burlan de mi físico & $\mathbf{0 . 6 0}$ & -0.46 & 0.348 \\
& Me dejan solo en actividades de equi- & $\mathbf{0 . 5 4}$ & -0.53 & 0.365 \\
R19 & po & $\mathbf{0 . 5 3}$ & -0.31 & 0.268 \\
R6 & Me ignoran & $\mathbf{0 . 4 9}$ & -0.42 & 0.297 \\
R11 & Me esconden cosas & $\mathbf{0 . 4 1}$ & -0.37 & 0.244 \\
R12 & Me roban cosas & 0.54 & $\mathbf{- 0 . 7 4}$ & 0.489 \\
R14 & Me amenazan para que sienta miedo & & \\
& Me obligan a hacer cosas que no quie- & 0.41 & $\mathbf{- 0 . 7 2}$ & 0.413 \\
R15 & ro & 0.31 & $\mathbf{- 0 . 5 9}$ & 0.292 \\
R16 & Me tocan mis partes íntimas & 0.51 & $\mathbf{- 0 . 5 9}$ & 0.393 \\
R18 & Me sacan del grupo de amigos & 0.55 & $\mathbf{- 0 . 5 9}$ & 0.396 \\
R13 & Me pegan &
\end{tabular}

Nota. Las cifras en "negritas" indican las cargas factoriales más altas.

En un tercer momento se llevó a cabo el análisis factorial confirmatorio. En la escala de agresores se descartó el reactivo 48 y en la escala de víctimas el reactivo 12, por no cumplir con los ajustes de medidas que se enuncian a cotinuación. Eliminando estos ítem, las escalas presentaron parámetros de confiabilidad adecuados, representados en los siguientes estadísticos de bondad de ajuste: GFI (Índice de Bondad de Ajuste) y AGFI (Bondad Ajustado de Índice de Ajuste) >.90 (Manzano y Zamora, 2009), CFI (Índice de Ajuste Comparado) $>.90$ (Bentler, 1990), SRMR (Estandarizado Root Mean Residual) <.05 (Steiger y Lind, 1980), y RMSEA (Error de Aproximación Cuadrático Medio) <.08 (Hair, Anderson, TathaM, y Black, 2010).

Para los ajustes de $\mathrm{X}^{2 / \mathrm{gl}}$ (probabilidad de significación estadística de Chi-cuadrado) los ajustes de los factores de ambas escalas no se encuentran dentro de los estándares establecidos ya que es muy sensible a muestras de análisis muy grandes y el ajuste de RMSEA se ubica en los límites de tolerancia; sin embargo, en los modelos en conjunto se observa un ajuste adecuado (véase en las Tablas 6 y 7 ). 
Tabla 6. Análisis factorial confirmatorio: Indicadores de bondad de ajuste de la escala de agresores $(N=2345)$

\begin{tabular}{lccccccccc}
\hline Subescala & $X^{2}$ & $g l$ & $X^{2 / g l}$ & GFI & AGFI & CFI & SRMR & RMSEA \\
\hline $\begin{array}{l}\text { Factor 1 } \\
\text { bal/Físico) }\end{array}$ & (Ver- & 236 & 20 & 11 & 0.97 & 0.95 & 0.95 & 0.03 & 0.06 \\
$\begin{array}{l}\text { Factor 2 } \begin{array}{l}\text { 2 } \\
\text { xual/Relacional) }\end{array} \\
\text { (Se- }\end{array}$ & 23 & 2 & 11 & 0.99 & 0.97 & 0.98 & 0.02 & 0.06 \\
\hline
\end{tabular}

Tabla 7. Análisis factorial confirmatorio: Indicadores de bondad de ajuste de la escala de víctimas $(N=2345)$

\begin{tabular}{lcccccccc}
\hline Subescala & $X^{2}$ & $g l$ & $X^{2 / g l}$ & GFI & AGFI & CFI & SRMR & RMSEA \\
\hline $\begin{array}{l}\text { Factor 1 } \\
\text { (Verbal/Relacional) }\end{array}$ & 165 & 14 & 11 & 0.98 & 0.95 & 0.96 & 0.03 & 0.06 \\
$\begin{array}{l}\text { Factor 2 } \\
\text { Física/Social) }\end{array}$ & 104 & 5 & 20 & 0.98 & 0.94 & 0.96 & 0.03 & 0.09 \\
\hline
\end{tabular}

Por último, se procedió a realizar un análisis del modelo en el que se incluyeron ambos factores para cada escala, resultando satisfactorios en rangos de normalidad los índices de bondad de ajuste de ambos modelos, lo que permite confirmar la estructura de validez y fiabilidad de las escalas (véase en Figuras 1 y 2). 


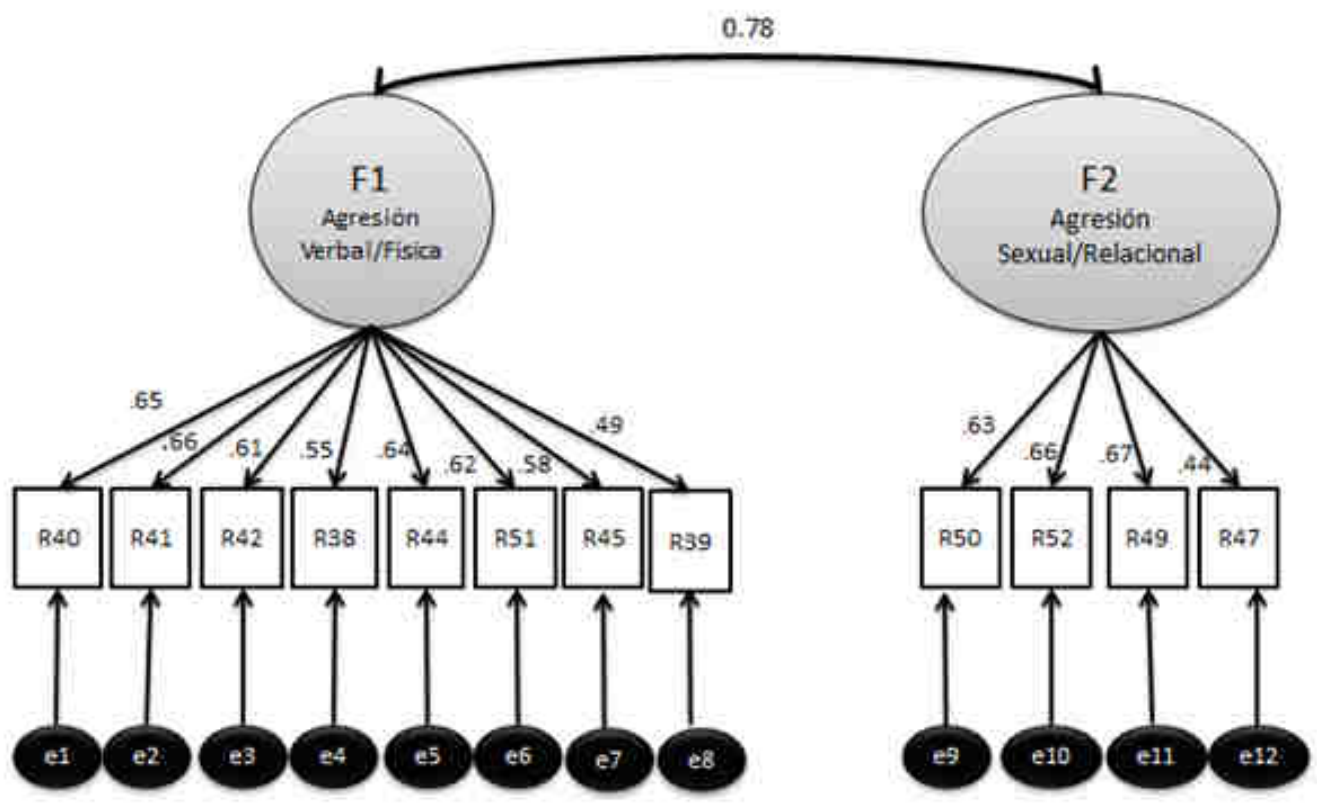

Indicadores de bondad de ajuste del modelo: $\mathrm{X}^{2}=673 ; \mathrm{gl}=53 ; \mathrm{X}^{2} / \mathrm{gl}=12 ; \mathrm{GFI}=0.95$; CFI $=0.91 ;$ RMSEA $=0.07 ; \mathrm{AGFI}=0.93 ; \mathrm{SRMR}=0.04 ; \alpha=0.84$

Figura 1. Análisis factorial confirmatorio para la escala de agresores

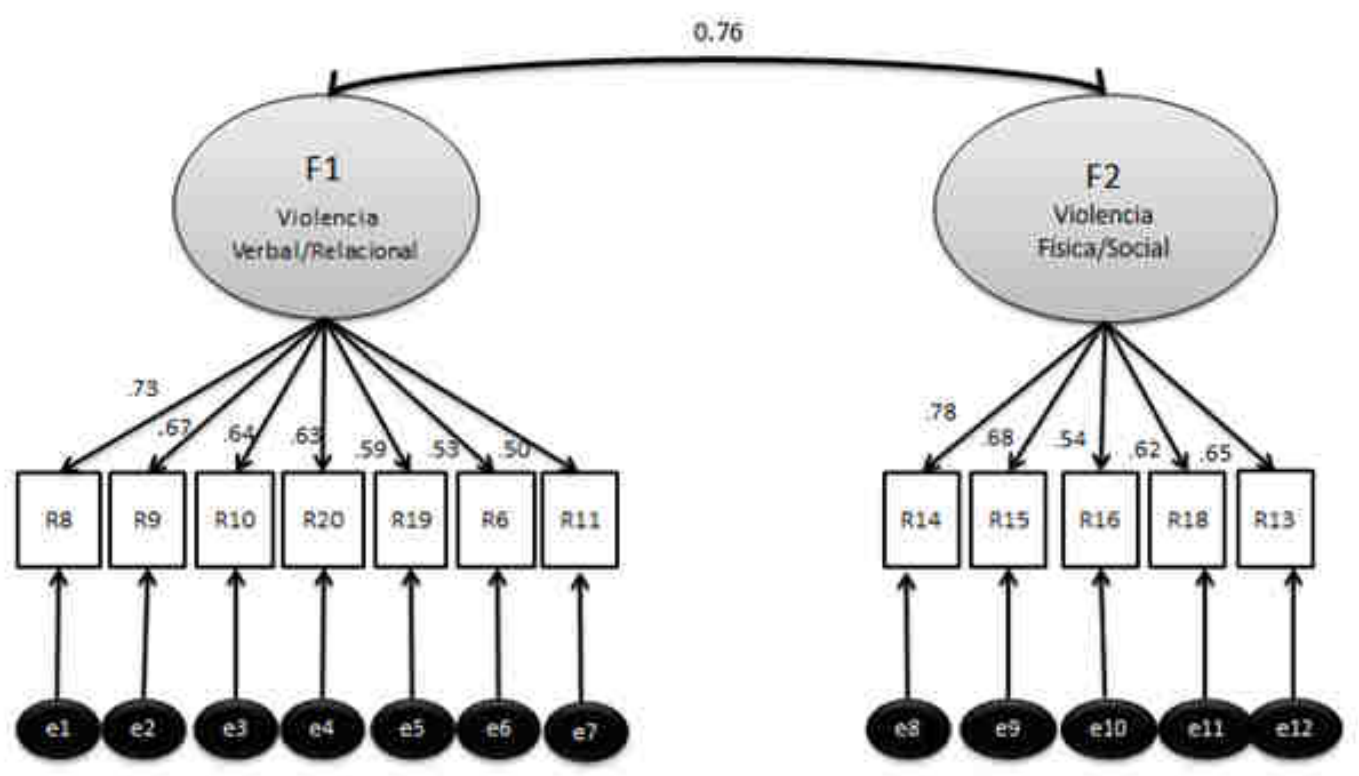

Indicadores de bondad de ajuste del modelo: $\mathrm{X}^{2}=908 ; \mathrm{gl}=53 ; \mathrm{X}^{2} \mathrm{gl}=17 ; \mathrm{GFI}=0.93$; CFI $=0.90$; RMSEA $=0.08 ;$ AGFI $=0.90 ;$ SRMR $=0.05 ; \alpha=0.85$

Figura 2. Análisis factorial confirmatorio para la escala de víctimas 
En la figura 1 se muestran los factores de agresión verbal/Física y el de sexual relacional de la Escala de agresores; y en la figura 2 los factores de violencia verbal/relacional y el de violencia física/social de la Escala de Víctimas. En ambas figuras se destacan correlaciones entre sus factores, encontrándose en ellos rangos altos de relación entre sí.

\section{Discusión y Conclusiones}

Es conocida la relación de dominio y sumisión que existe en el agresor y la víctima de violencia entre pares, siendo esta relación un factor de riesgo que hace sostenida en el tiempo dicha violencia y que puede traer consecuencias negativas tanto para quien la ejerce, como para quien la sufre. En ese sentido, el objetivo de esta investigación ha sido validar la escala de víctimas y agresores y sus roles asociados al acoso escolar, midiendo las percepciones de los alumnos al protagonizar los roles de víctimas y agresores, de tal forma que esto nos permita identificar dichos roles y elaborar medidas de prevención.

Con los resultados obtenidos encontramos que la escala de víctimas de Valdés (2013), que originalmente dimensiona los tipos de violencia recibidos en: violencia física, social, psicológica, sexual y cyber-bullying; luego de los análisis y al eliminarse los reactivos: 7 "no me dejan participar", 17 "me obligan a tocarles sus genitales", y 12 "me roban mis cosas" por no cumplir con los estándares aceptados según los criterios de outfit (normalidad), se dimensionó en dos factores: "violencia verbal", en donde se unieron las dimensiones cyber-bullying y psicológica, y por otra parte la dimensión de "violencia física" que conjuga la violencia sexual.

Por otro lado, la escala de agresores de Ortega y del Rey (2005) tiene la estructura de los tipos de agresiones en: física, social, psicológica, sexual y cyber-bullying, en la cual, después del análisis se eliminaron los reactivos: 48 "obligar a un compañero a que te toque tus partes íntimas", 43 "robarles" y 46 "obligarles a hacer cosas que no quieren" por no cumplir con los estándares aceptados según los criterios de outfit (normalidad); y se dimensionó en solo dos factores: "agresión verbal" conjugando las agresiones sociales y psicológicas; y por otra parte la "agresión fisica" en el que se integra el cyber-bullying. 
Por lo anterior, se puede observar que en ambas escalas coinciden la eliminación de reactivos que tienen que ver con: violencia física hacia las pertenencias de otros; violencia sexual relacionada a tocar las partes íntimas de otro; y violencia social referida al sometimiento de hacer o dejar de hacer algo. Esto puede deberse a la cultura del silencio ante este tipo de situaciones que son encubiertas por los mismos alumnos, tanto de quien lo hace como quien lo recibe o ve, ya que sí existen casos de estos tipos de violencia en las escuelas, pero suelen ser ocultados por factores como vergüenza y miedo, aun más en la etapa de la adolescencia (entre 12 y 17 años de edad) en la que se encuentran los estudiantes de nivel secundaria. Este fenómeno de la "ley del silencio" Ortega (1998) lo define como una obligación ejercida a manera de presión social entre los alumnos para callar e ignorar las situaciones de violencia (aplicada a todos los roles), por lo que, a la par de su omisión, se vuelven complices y copartícipes de dicha situación. De esta forma el agresor se siente aprobado debido al consentimiento implícito que se transmite por la conducta de omisión; por su parte, la víctima puede sentir que no solo esta siendo violentada por el agresor sino por sus compañeros, que, en lugar de ayudarle optan por no decir o hacer algo en pro de apoyarle; y los espectadores, al no intentar parar la situación, puede llegar a interpretarse como "estar del lado del agresor", es decir "si no ayudas a la víctima, ayudas al agresor", donde sus conductas tienen una gran influencia entre los actos de violencia perpetrados por el agresor hacia la víctima, donde el hecho de observar dicha situación es reforzante para el agresor, aunque pudiera ser que los observadores estuvieran de parte de la víctima, pero que se acogen a la ley del silencio para no salir igualmente lastimadas o perjudicadas en ese momento o en el futuro. Sin embargo, la ley del silecio dificulta a las autoridades poder identificar la relación que se esconde tras las agresiones, donde en ocasiones, de no ser violencia evidentemente física, por desgracia se hace menos notable para la oportuna detección. Esto mismo señala Carozzo (2015), indicando que la complicidad es un lazo de adhesión y cohesión en haras de la socialización entre ellos mismos, por lo que denunciar las situaciones de violencia significaría romper con las normas sociales no escritas entre los mismos alumnos, y por ende se entendería como una ruptura socioescolar.

La socialización escolar puede llegar a crear las bases para una sana convivencia, sin embargo, habría que plantear estrategias y habilidades en pro de fomentar la de denuncia, promoviendo la educación solidaria, de respeto y equidad, donde se desalienten las conductas transgresoras y se fomenten los ambientes y climas escolares democráticos-participativos y pacíficos. Por ello, es que las escalas anteriormente presentadas, además de cumplir adecua- 
damente con los parámetros y propiedades psicométricas, pueden ayuda a evaluar la presencia de conductas de agresión física, verbal, psicológica, Cyberbullying en las escuelas secundarias públicas del Estado. Por lo que se recomienda investigar a través de variables individuales, familiares y escolares las relaciones Agresor-Víctima-Espectadores, para generar a través de programas de prevención de la violencia y promoción de valores un ambiente escolar en el cual los estudiantes convivan de manera pacifica (Doménech e Íñiguez, 2002).

\section{Referencias}

Albaladejo, N. (2011). Evaluación de la violencia escolar en educación infantil y primaria (Tesis doctoral), Universidad de Alicante. España

Bentler, P. (1990). Comparative Fit Indexes in Structural Models. Psychological Bulletin, 107 (2), pp. 238-46.

Bourdie, P. y Passerson, J. (1996). La Reproducción, Elementos para una teoría del sistema de enseñanza. Madrid: Editorial Popular.

Carozzo, J. (2015). Los espectadores y el código del silencio. Revista Espiga, 14(29), 1-8. Recuperado el 10 de Enero del 2015 en: http://investiga.uned.ac.cr/revistas/index.php/espiga/article/view/948

Chávez, C. y Saade, A. (2009). Procedimientos básicos para el análisis de reactivos. Cuaderno técnico 8. CENEVAL: México

González-Montesinos, M. (2008). El análisis de reactivos con el Modelo Rasch. Manual técnico A. Universidad de Sonora-Instituto Nacional para la Evaluación de la Educación. Recuperado el 5 de Enero del 2015 en: http://www.winsteps.com/a/recursos-offline.pdf

Hair, J., Anderson, R., TathaM, R., \& Black, W. (2010). Multivariate Data Analysis: A Global Perspective (7th ed.). Upper Saddle River, NJ: Pearson Prentice-Hall.

Hair, J., Anderson, R., Tatham, R. y Black, W. (1999). Análisis Multivariante. $5^{\mathrm{a}}$ ed. Prentice Hall: Madrid, España.

Kulig, J., Hall, B. y Kalischuk, R. (2008). Bullying perspectives among rural youth: a mixed methods approach. Rural and remote health, 8(923), 1-11.

Manzano, A. y Zamora, S. (2009). Sistema de ecuaciones estructurales: una herramienta de investigación, Cuaderno técnico 4. Centro Nacional de Evaluación para la educación superior, A.C. (Ceneval). México, DF.

Ortega, R. (1998). La convivencia escolar: qué es y cómo abordarla. Consejería de Educación y Ciencia. Junta de Andalucía. 
Steiger, J, y Lind, J (1980). Statistically-based tests for the number of common factors. Paper presented at the annual Spring Meeting of the Psychometric Society in Iowa City.

Wright, B., y Linacre, J. (1994). Reasonable mean-square fit values. Rasch Measurement: Transactions of the Rasch Measurement SIG, 8(3), 370. 